\title{
TW Dra: NRP mode identification with FAMIAS
}

\author{
H. Lehmann, ${ }^{1}$ A. Tkachenko, ${ }^{1}$ and D. E. Mkrtichian ${ }^{2,3}$ \\ 1 Thüringer Landessternwarte Tautenburg, Germany \\ ${ }_{2}$ ARCSEC, Sejong University, Seoul, Korea \\ ${ }^{3}$ Astronomical Observatory, Odessa National University, Ukraine
}

\begin{abstract}
TW Dra is an Algol type system where the primary shows $\delta$ Sct oscillations. Time series of high-resolution spectra taken at the Thüringer Landessternwarte Tautenburg (TLS) show a complex pattern of moving bumps across the line profiles, indicating a rich spectrum of high-degree non-radial pulsation modes. After the reductions to remove the signatures of the second and third components we analyze the line profiles using the FAMIAS program to derive the oscillation frequencies and to identify the pulsation modes. We describe the way in which mode identification can be done by FAMIAS and present first results.
\end{abstract}

Individual Objects: TW Dra

\section{Introduction}

The Algol type system TW Dra (A5V+K0III) was detected to be a member of the new class of oEA stars (i.e. Algol type systems where the mass accreting primary shows $\delta$ Scuti oscillations; Mkrtichian et al. 2002, 2004) by Kusakin et al. (2001). Photometrically, it is one of the best investigated oEA stars. Here we present a first spectroscopic investigation of non-radial pulsation (NRP) modes using the program package FAMIAS (Zima 2008). Time series of high-resolution spectra were taken in 13 consecutive nights with the Coudé-echelle spectrograph at the $2 \mathrm{~m}$ telescope at TLS and in two nights with the BOES spectrograph at the $1.8 \mathrm{~m}$ telescope at Bohyunsan Optical Astronomy Observatory (BOAO).

The analysis is complicated by the fact that TW Dra is a member of a visual binary and that the obtained spectra partly contain light contributions from the third component. In preparation of the investigation of NRP modes we derived first a precise orbital solution using KOREL (Hadrava 2004). From this we obtained the masses $M_{1}=2.13 \mathrm{M}_{\odot}, M_{2}=0.89 \mathrm{M}_{\odot}$, and the separation $a=12.11 \mathrm{R}_{\odot}$ of the components of the close system. KOREL delivered also the disentangled spectra of all three components from which we derived important parameters of the primary like $v \sin i=47 \mathrm{~km} \mathrm{~s}^{-1}$, microturbulence of $2.9 \mathrm{~km} \mathrm{~s}^{-1}$ and a slight metal overabundance. Values of $T_{\text {eff }}=8150 \mathrm{~K}$ and $\log g=3.88$ were taken from the photometric solution. To gain a higher $\mathrm{S} / \mathrm{N}$ we applied least squares deconvolution (LSD, Donati et al. 1997) to the spectra and removed the signatures of the second and third components in fitting the disentangled spectra shifted by the orbital radial velocity to the composite LSD line profiles. All these procedures and results are described by Lehmann et al. (2008). 


\section{Frequency analysis of oscillation modes and mode identification}

We did a multiple frequency search based on successive pre-whitening of the data as well in the line profile moments as in the profiles itself by using the pixel-by-pixel method (calculation of the Fourier spectrum of the temporal variation of each pixel-value across the line profile). Here we restricted the used dispersion range as close as possible to the inner line core where the high-frequency contributions dominate while the variations in the outer wings are mainly determined by low frequencies arising from orbital motion and imperfect removal of the second and third components' contributions. By using the Fourier method, three oscillations in the high-frequency domain were found: $F_{1}=22.90 \mathrm{~cd}^{-1}, F_{2}=14.05 \mathrm{~cd}^{-1}$, and $F_{3}=24.72 \mathrm{~cd}^{-1}$, in difference to the values of $17.99 \mathrm{~cd}^{-1}$ or $18.95 \mathrm{~cd}^{-1}$ that were detected by Kusakin et al. (2001) or Kim et al. (2003) photometrically. Also a frequency search in the first three LSD profile moments did not reveal the photometric values, but in the second order moment we found a narrow doublet centred at this place. Instead we found two frequencies of $f_{1}=20.29 \mathrm{~cd}^{-1}$ and $f_{2}=25.26 \mathrm{~cd}^{-1}$ or their $1 \mathrm{~cd}^{-1}$ or $2 \mathrm{~cd}^{-1}$ aliases in all of the three moments. The $1 \mathrm{~cd}^{-1}$ alias of $f_{1}$ is also the strongest mode detected in the photometry obtained by E. Rodriguez at Sierra Nevada Observatory in three nights in March 2008 where the $f_{2}$ mode can be seen as well.

Based on the multiple frequency model obtained from the Fourier analysis of the line profiles, we tried to identify the modes by using the Fourier Parameter Fit (FPF) method of FAMIAS. The model included the three oscillation frequencies $F_{1}$ to $F_{3}$ as well as some lowfrequency contributions. Basic stellar parameters were fixed to the above mentioned values. In a first step we derived the equivalent width and RV zero point of the mean profile. After fixing the pulsation phases found from a free parameter search, we used the grid-mode search to optimize $I$ and $m$ quantum numbers together with the remaining free parameters $v \sin i$, intrinsic width and pulsation velocity. Unfortunately, we did not find an unique solution and present here the solutions having lowest chi-square: $(I, m)=(7,5),(7,7),(8,8)$ for $F_{1} ;(7,5)$, $(8,6),(8,8)$ for $F_{2} ;(10,6),(11,7),(11,9),(11,11)$ for $F_{3}$.

\section{Conclusions}

Using the pixel-by-pixel method of FAMIAS, three oscillation modes in the high-frequency domain have been detected which are all different from those found in the photometry before. We found no unique solution from mode identification and can present here only different possible combinations of $I$ and $m$. All these combinations include only high-degree $I$ modes. We have also detected two pulsation modes in the first three moments which are in agreement with those found in the photometry. We assume that from the line profile moments and from photometry we will find only low degree modes whereas the high-degree I modes can be found only with the pixel-by-pixel method.

In the near future we want to improve the removal of the secondary and third components with the aim to use broader line regions in the analysis, to analyze the suspected low-degree modes, and to model the eclipse phases with SHELLSPEC (Budaj et al. 2005) to analyze line profiles variations by eclipse mapping (Gamarova et al. 2003).

Acknowledgments. Mode identification was obtained with the software package FAMIAS developed within the FP6 European Coordination Action HELAS (http://www.helas-eu.org/).

\section{References}

Budaj, J., Richards, M. T., \& Miller, B. 2005, ApJ, 623, 411

Donati, J.-F., Semel, M., Carter, B. D., et al. 1997, MNRAS, 291, 658

Gamarova, A. Yu., Mkrtichian, D. E., Rodriguez, E., et al. 2003, ASP Conf. Ser., 292, 369 
Hadrava, P. 2004, Publ. Astron. Inst. ASCR, 92, 15

Kim, S.-L., Lee, J. W., Kwon, S.-G., et al. 2003, A\&A, 405,231

Kusakin, A. V., Mkrtichian, D. E., \& Gamarova, A. Yu. 2001, IBVS, 5106

Lehmann, H., Tkachenko, A., Tsymbal, V., \& Mkrtichian, D. E. 2008, CoAst, 157, 332

Mkrtichian, D. E., Kusakin, A. V., Rodriguez, E., et al. 2004, A\&A, 419, 1015

Mkrtichian, D. E., Kusakin, A. V., Gamarova, A. Yu., \& Nazarenko, V. 2002, ASP Conf. Ser., 259, 96

Zima, W. 2008, CoAst, 155, 17

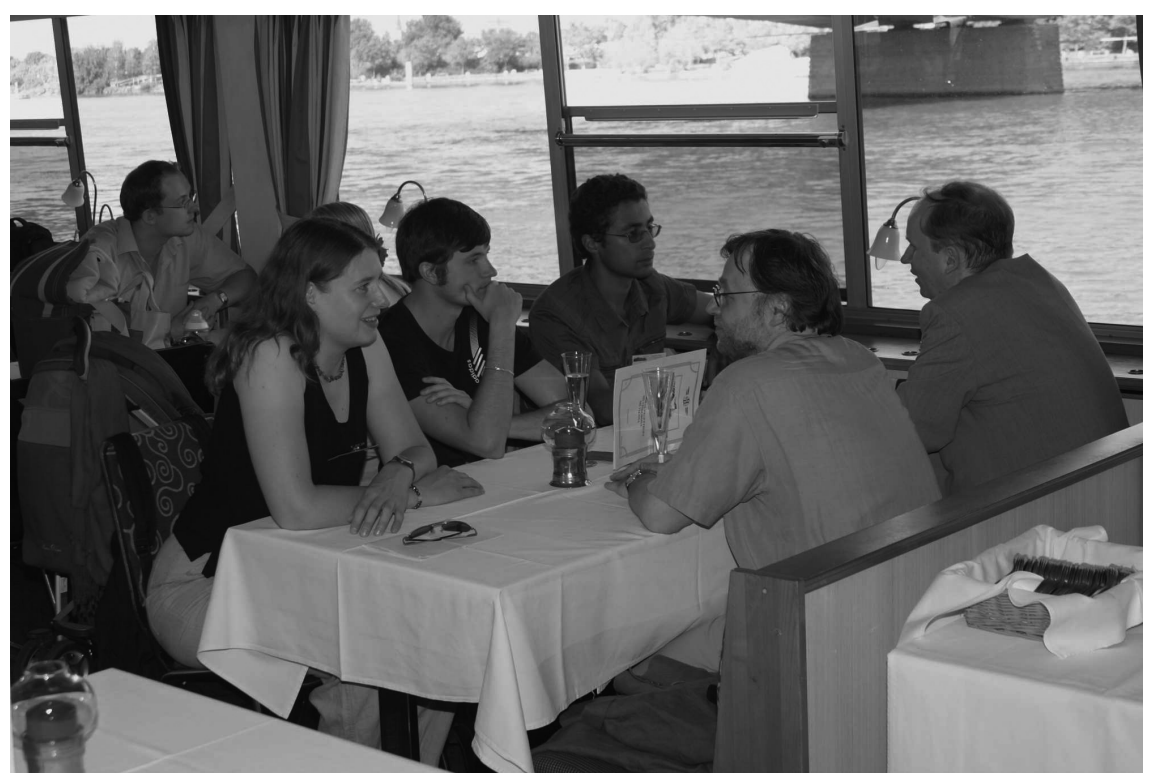

Excursion on the Danube river. 